\title{
Téoros
}

Revue de recherche en tourisme

\section{Des végétaux pour voyager}

Les avantages et les inconvénients des contreparties de la fixation du carbone par la séquestration dans les forêts

\section{Julianna Priskin et Renae Nicole Stenhouse}

Volume 26, numéro 3, automne 2007

URI : https://id.erudit.org/iderudit/1071009ar

DOI : https://doi.org/10.7202/1071009ar

Aller au sommaire du numéro

Éditeur(s)

Université du Québec à Montréal

ISSN

0712-8657 (imprimé)

1923-2705 (numérique)

Découvrir la revue

Citer cette note

Priskin, J. \& Stenhouse, R. N. (2007). Des végétaux pour voyager : les avantages et les inconvénients des contreparties de la fixation du carbone par la séquestration dans les forêts. Téoros, 26(3), 68-71.

https://doi.org/10.7202/1071009ar d'utilisation que vous pouvez consulter en ligne. 


\section{Des végétaux pour voyager}

\section{Les avantages et les inconvénients des contreparties de la fixation du carbone par la séquestration dans les forêts}

\section{Julianna Priskin et Renae Nicole Stenhouse}

Les voyageurs tout comme l'industrie du tourisme consomment d'importantes quantités de produits dérivés des combustibles fossiles, notamment pour les activités de transport aérien et routier (Kelly et Williams, 2007). Des gaz à effet de serre (GES) associés à cette consommation sont libérés dans l'atmosphère, contribuant aux changements climatiques d'origine anthropogénique, ce qui devrait continuer d'avoir des incidences à la fois positives et négatives sur les destinations (UNWTO, 2007). Les voyageurs et l'industrie du tourisme sont donc appelés à participer à la recherche de solutions d'atténuation (Burns et Bibbings, 2007). Les mécanismes de marché, dont l'achat volontaire de crédits de carbone ${ }^{1}$ que l'on se procure auprès de divers organismes, peuvent aider à assurer un voyage à bilan carbone neutre (Priskin, 2007a ; 2007b).

Le voyage à bilan carbone neutre représente plus qu'une simple tendance chez ceux qui ont une conscience écologique (Anonyme, 2007a: 3-30; Anonyme, 2007b : 61). Cela dit, les mécanismes volontaires de compensation des émissions de carbone apportent autant de questions que de réponses aux émissions polluantes de GES. Présentement, cette tendance soulève des questions liées aux aspects suivants :

- les marchés réglementés et non réglementés de carbone;

- l'efficacité des divers programmes de compensation des émissions de carbone;

- la crédibilité des organismes qui vendent des crédits de carbone à l'intérieur de programmes de compensation ;

- le peu de conscientisation des consommateurs concernant les mécanismes de compensation de carbone (Gössling et al. 2007 ; Heughebaert, 2006 ; Kollmuss et Bowell, 2007).

Une tendance générale se dessine parmi les voyageurs et le secteur du tourisme (allant des hôtels aux compagnies aériennes) à investir dans des projets de plantation d'arbres pour traiter les émissions de GES (Sustainable Travel International, 2007). Si l'importance de ces mesures destinées à neutraliser les émissions doit être reconnue, la plantation d'arbres, néanmoins, soulève beaucoup de controverses (Barker, 2006). Le présent article traite des enjeux associés à l'efficacité des programmes de compensation des émissions de carbone par la plantation d'arbres et propose une vision équilibrée de ce sujet controversé dans le but de le faire mieux connaître.
En dépit de la complexité apparente des changements climatiques d'origine anthropogénique, le problème principal concerne le déséquilibre du cycle du carbone (Watson, 2001). Le carbone et les autres gaz à effet de serre forment normalement une composante du cycle naturel des échanges entre la surface terrestre et l'atmosphère. Cependant, les émissions de carbone dues aux différents types d'activité humaine se sont accumulées dans l'atmosphère à des taux supérieurs au cycle naturel. Ce phénomène bouleverse les processus écologiques et est susceptible de contribuer au réchauffement planétaire (UNFCC, 2007). Les solutions aux changements climatiques provoqués par les activités humaines résident dans la réduction de la quantité excessive de carbone et des autres GES stockés dans l'atmosphère, pour faire en sorte que le cycle naturel du carbone sur terre arrive à un équilibre qui, selon les scientifiques, n'entraînera pas de conséquences (IPCC, 2007).

Réduire directement les émissions de GES n'est pas toujours une option viable pour l'industrie du voyage et du tourisme. Des mesures de mitigation supplémentaires, par exemple la compensation pour la pollution, sont donc essentielles. Parmi les programmes de compensation existants, notons les investissements dans les projets d'énergie renouvelable visant à éliminer la dépendance à l'égard des ressources de combustibles fossiles; le soutien aux projets technologiques pour parvenir à une utilisation plus efficace des ressources ; l'investissement dans des projets qui contribuent à l'absorption du carbone de l'atmosphère. Ces projets de puits de carbone permettent la séquestration ou le stockage des émissions de carbone dans des réservoirs géologiques ou biologiques (UNEP, 2006 ; World Resources Institute, 2007). Les projets de puits de carbone biologiques comprennent notamment la plantation d'arbres qui, au cours de leur vie, absorbent le carbone atmosphérique.

\section{Les avantages : comment les arbres peuvent réduire les gaz à effet de serre}

Il est évident que les forêts ont un rôle inestimable dans la réduction à long terme des GES (UNFCC, 2007 ; IPCC, 1996). La croissance des arbres (et des autres organismes qui se nourrissent par photosynthèse) permet l'absorption ou la séquestration du carbone de l'atmosphère. Le sol dans lequel ils vivent participe aussi à la séquestration du carbone. La quantité absorbée peut varier selon la localisation géographique, le climat, la géologie, la topographie, le type de sol, les espèces arborescentes et la durée de la plantation (Baral et Guha, 2004). La gestion de la plantation est essentielle pour assurer la survie des semences et lutter contre les maladies et les 
incendies et ainsi optimiser la séquestration du carbone. Si ces conditions se maintiennent et les arbres ne sont pas récoltés, le processus de séquestration s'arrêtera après 100 ou 200 ans, quand les arbres auront atteint leur maturité. Ceux-ci vont éventuellement mourir de cause naturelle et, en se décomposant, leur capacité de séquestration du carbone diminuera plus rapidement.

La plantation d'arbres peut contribuer à résoudre d'autres problèmes environnementaux; elle peut contribuer à :

$$
\begin{aligned}
& \text { - requalifier les espaces dégradés, } \\
& \text { - rétablir un niveau d'équilibre des eaux et réduire la salinité, } \\
& \text { - soutenir la conservation de la biodiversité, } \\
& \text { - réaliser des recettes ainsi que le développement économique. }
\end{aligned}
$$

Pour que les végétaux soient considérés comme étant des puits de carbone, certaines conditions doivent être remplies. Dans le cas de l'Australie, par exemple, les arbres doivent atteindre 2 mètres de haut à maturité, avoir un taux de couverture de la canopée de $20 \%$ et faire partie d'un peuplement forestier d'une surface supérieure à 0,2 hectare (Australian Greenhouse Office, 2006). Certaines espèces d'arbres et d'arbustes en plus des vergers sont admissibles, alors que les vignobles et les prairies sont exclus.

De manière générale, les coûts de mise en œuvre de puits de carbone forestiers sont 90 \% moins élevés que l'élaboration et l'implantation de technologies écoénergétiques pour des projets de compensation des émissions de carbone (van Kooten et al., 2004). En outre, le fait d'acheter des crédits de carbone dans le contexte de projets de reboisement véhicule une image positive, verte et propre des entreprises et des individus.

\section{Les mises en garde : pourquoi les arbres ne peuvent pas résoudre les problèmes des gaz à effet de serre}

Un nombre d'incertitudes persiste concernant la séquestration du carbone dans les puits forestiers. D'abord, il y a l'enjeu de la permanence. Le potentiel des projets forestiers à emmagasiner le carbone s'estompe quand la forêt disparait (Smith et Scherr, 2003). Lorsque les plantations à courte ( $<10$ ans) ou à moyenne ( $<15$ ans) rotation périssent, le carbone est relâché dans l'atmosphère et les émissions d'un client (qui a acheté des crédits de carbone) ne sont pas réellement compensées (Kirschbaum, 2000). Par ailleurs, le carbone séquestré à plus long terme par la plantation d'arbres peut être relâché de manière intentionnelle (par exemple par la récolte) ou non intentionnelle (sècheresse, incendie, maladie) (Bäckstrand et Lövbrand, 2006 ; Maréchal et Hecq, 2006). L'absence de moyens de gestion peut écourter la vie des arbres et rendre le puits moins « étanche» (Barker, 2006). Certains critiques laissent entendre que le territoire disponible pour accueillir les plantations est insuffisant en raison des conditions biogéographiques (par exemple les paysages exposés au risque d'incendie) ou des risques liés au pays (par exemple, l'instabilité politique, l'insécurité du régime foncier) (Benitez et al., 2007 ; Jindal, 2006). Par ailleurs, R. Barker (2006) et F. Pearce (2002) font état d'études qui montrent que défricher le sol avant de planter a pour effet de relâcher dans l'air le dioxyde de carbone entreposé dans la terre ainsi que la matière organique et ce, possiblement à un niveau plus élevé que la quantité de dioxyde de carbone qui avait été absorbée dans les arbres au cours des dix premières années de la plantation.

La rentabilité et la valeur écologique d'une plantation comme puits de carbone varient selon les espèces d'arbres plantées. Les agroforêts sont composées d'une multitude d'espèces d'arbres et ont une capacité de stockage du carbone accrue, une biodiversité plus riche que les monocultures et plus de chances d'avoir des répercussions positives sur l'économie locale (Kirby et Potvin, 2007). Les plantations en monoculture composées d'essences non indigènes (par exemple les eucalyptus ou les pins) ne mettent pas en valeur la biodiversité, se prêtent mal à l'établissement d'habitats, perturbent le cycle hydrologique, augmentent l'utilisation de produits chimiques et de pesticides et peuvent contribuer à l'augmentation de l'acidité du sol (Bäckstrand et Lövbrand, 2006 ; Barker, 2006).

En raison de leurs prétendues retombées sur le développement durable, l'idée que les pays développés établissent des plantations de puits de carbone dans les pays en développement est bien accueillie (Jindal, 2006). Ce genre de projet a pourtant suscité des critiques. Des études de cas menées au Mexique montrent que les collectivités et les producteurs à revenu moyen profiteraient davantage des marchés de carbone forestiers, alors que les membres moins présents et puissants de la collectivité sont exclus des processus de prise de décisions et des bénéfices (Brown et Corbera, 2003). Les projets de plantation de grande envergure ont un bon rapport coût-efficacité avec des faibles coûts de transaction, les rendant ainsi attrayants aux yeux des promoteurs. Cependant, ils constituent une menace pour les collectivités locales qui risquent d'être déplacées, de voir leur accès aux terres ancestrales interdit ou d'obtenir une compensation dérisoire (Bäckstrand et Lövbrand, 2006 ; Smith et Scherr, 2003).

Une fois la forêt implantée, la mesure du carbone absorbé pendant toute la durée du projet est un processus complexe (Richards et Anderson 2001). De nombreux facteurs (comme ceux décrits plus haut) influencent la capacité d'une forêt à séquestrer le carbone, à un point tel qu'il est difficile d'estimer avec précision la quantité totale de carbone séquestré par un projet ou le coût de la séquestration (Maréchal et Hecq, 2006 ; Smith et Scherr, 2003 ; van Kooten et al., 2004). De nombreuses entreprises œuvrant dans le secteur des crédits de carbone utilisent leur propre « formule " pour estimer la valeur d'une empreinte carbonique, ce qui a pour résultat que la distance parcourue en avion, l'empreinte carbonique, le nombre d'arbres nécessaires pour compenser la pollution générée par le vol et le prix de ces arbres peuvent varier considérablement (Barker, 2006).

Il existe présentement un vide réglementaire en ce qui concerne les crédits pour les puits de carbone forestiers. La vérification des projets de séquestration du carbone est prise en charge par des tiers sélectionnés par l'entreprise qui vend ces crédits. Par conséquent, des entreprises, motivées par l'opportunisme, peuvent exagérer ou gonfler les bénéfices escomptés du projet de réduction des émissions (Richards et Anderson, 2001). La pratique de l'autorégulation est la plus répandue aujourd'hui. On peut se demander alors, dans le cas d'un projet de plantation d'une durée de cent ans où des profits sont réalisés dès la première année, comment une entreprise sera-t-elle incitée à investir dans la gestion à long terme de la plantation? 
La dernière mise en garde émise au sujet de la séquestration du carbone est que celle-ci ne se traduit pas directement par une réduction de la dépendance des combustibles fossiles. Elle permet de compenser les émissions de dioxyde de carbone plutôt que de réduire la pollution à sa source. Compte tenu que le boisement et le reboisement ne contribuent pas à la diminution des émissions, bon nombre d'organisations ne vendent pas de crédits de carbone séquestré. Ces dernières préfèrent mettre l'accent sur des projets d'énergie renouvelable et d'efficacité énergétique, lesquels visent à appuyer une transition de la situation de dépendance actuelle envers les combustibles fossiles. Le Gold Standard constitue la seule norme indépendante dans le marché volontaire du carbone qui exige la complémentarité, la mobilisation de partenaires locaux, la transparence et l'évaluation indépendante de chacun des projets (Gold Standard Foundation, 2007). Les plantations en sont exclues.

\section{Bien choisir les mesures en faveur de la séquestration du carbone}

Si les voyageurs s'engagent à devenir neutres en carbone en favorisant la séquestration du carbone, il serait judicieux qu'ils effectuent auparavant une vérification complète des diverses entreprises. Présentement, il n'existe pas de normes internationales, de réglementation ou de lignes directrices claires dans le domaine de la compensation volontaire d'émissions de carbone. Au Royaume-Uni, le département gouvernemental responsable de l'environnement, de l'alimentation et des affaires rurales a publié une ébauche de code de pratiques exemplaires (WBCSD, 2007). Avant que sorte la version définitive et que des règlements spécifiques à cette industrie soient adoptés, les clients de crédits de carbone devront eux-mêmes enquêter sur les différentes entreprises (tableau 1).

\section{Conclusion}

Grâce aux crédits de carbone, nous pouvons continuer de dépendre des combustibles fossiles pour nos activités de voyage sans nous sentir coupables. La séquestration du carbone ne suffit pas à atteindre les objectifs de réduction des gaz à effet de serre (Kirschbaum, 2000). À terme, réduire les émissions de GES dépend de la diminution de I'utilisation des combustibles fossiles. La solution aux changements climatiques requiert des changements sociaux et une progression vers l'énergie verte dans tous les secteurs. Plus nous comptons sur les puits de carbone pour compenser les GES à court terme, plus l'essor des sources d'énergie alternative est freiné, rendant les objectifs de réduction des émissions atmosphériques encore plus difficiles à atteindre (idem, 2000). II faudrait que les puits de carbone soutiennent une telle révolution de l'énergie alternative plutôt que la remplacer ou la freiner.

Étant donné que les changements dans l'occupation du sol, principalement par la déforestation, expliquent $25 \%$ de toutes les émissions anthropogéniques de carbone, le plus grand effet bénéfique du stockage du carbone serait de prévenir la conversion des forêts en terres agricoles et en zones urbaines (Kirby et Potvin, 2007).

Pour diminuer son empreinte carbonique, une autre mesure consiste en l'achat de crédits de carbone certifiés dans des projets de compensation sans plantations, comme l'énergie renouvelable et les
Tableau 1

Une grille de contrôle pour la compensation responsable par des projets de séquestration du carbone

\begin{tabular}{|c|c|}
\hline Recherchez... & Évitez... \\
\hline $\begin{array}{l}\text { Une plantation permanente (<100 ans) } \\
\text { et protégée }\end{array}$ & $\begin{array}{l}\text { Les plantations à courte ( }<10 \text { ans) } \\
\text { ou moyenne ( }<15 \text { ans) rotation }\end{array}$ \\
\hline Des espèces indigènes & $\begin{array}{l}\text { Les plantes exotiques } \\
\text { (comme l'eucalyptus et les pins) }\end{array}$ \\
\hline Une plantation d'espèces variées & Les monocultures \\
\hline $\begin{array}{l}\text { Une plantation d'espèces feuillues } \\
\text { d'une longue durée de vie }\end{array}$ & $\begin{array}{l}\text { Une plantation d'espèces résineuses } \\
\text { ou d'une courte durée de vie (sauf si } \\
\text { la plantation est composée d'espèces } \\
\text { indigènes et adaptées) }\end{array}$ \\
\hline $\begin{array}{l}\text { Des moyens investis dans le soin } \\
\text { des arbres pour assurer leur survie et } \\
\text { longue vie }\end{array}$ & $\begin{array}{l}\text { Les coûts de gestion à la suite de } \\
\text { l'établissement de la plantation qui ne } \\
\text { sont pas inclus }\end{array}$ \\
\hline $\begin{array}{l}\text { La complémentarité (c'est-à-dire des } \\
\text { projets qui n'auraient pas pu se réaliser } \\
\text { sans votre achat) }\end{array}$ & $\begin{array}{l}\text { Un projet qui aurait pu être réalisé sans } \\
\text { le concours du client }\end{array}$ \\
\hline $\begin{array}{l}\text { Une entreprise qui remplace } \\
\text { immédiatement les arbres que vous } \\
\text { avez achetés et qui ont péri (par ex. à la } \\
\text { suite d'un incendie ou d'une maladie) }\end{array}$ & $\begin{array}{l}\text { L'absence de surveillance et de gestion } \\
\text { du suivi }\end{array}$ \\
\hline $\begin{array}{l}\text { Une organisation sans but lucratif avec } \\
\text { des frais généraux minimes }\end{array}$ & Des coûts de gestion et autres trop élevés \\
\hline $\begin{array}{l}\text { Des avantages environnementaux sup- } \\
\text { plémentaires (comme l'établissement } \\
\text { d'habitats) ou des retombées sur le } \\
\text { développement durable (par ex. une } \\
\text { véritable aide au développement } \\
\text { adaptée aux collectivités locales) }\end{array}$ & $\begin{array}{l}\text { Le défaut d'avantages } \\
\text { environnementaux supplémentaires ou } \\
\text { de retombées sociales }\end{array}$ \\
\hline $\begin{array}{l}\text { L'assurance que le crédit de carbone } \\
\text { que vous avez acheté est retiré } \\
\text { définitivement du marché }\end{array}$ & $\begin{array}{l}\text { Les crédits de carbone qui peuvent } \\
\text { être revendus (entraînant une double } \\
\text { comptabilisation) }\end{array}$ \\
\hline $\begin{array}{l}\text { La certification et la vérification par un } \\
\text { tiers indépendant }\end{array}$ & L'absence de vérification externe \\
\hline $\begin{array}{l}\text { La transparence - une entreprise qui } \\
\text { fournit des renseignements concernant } \\
\text { le projet, ses politiques, ses pratiques } \\
\text { et son système de vérification }\end{array}$ & $\begin{array}{l}\text { Des renseignements limités concernant } \\
\text { le projet }\end{array}$ \\
\hline $\begin{array}{l}\text { Un projet de plantation qui tient compte } \\
\text { des besoins et des connaissances des } \\
\text { populations locales }\end{array}$ & $\begin{array}{l}\text { Un projet de plantation qui ne favorise } \\
\text { pas l'adhésion par les collectivités } \\
\text { locales (qui n'ont aucun droit d'accès } \\
\text { et ne prennent pas de décision) }\end{array}$ \\
\hline
\end{tabular}

Source : auteures.

technologies «écoefficaces ». Cela contribue à l'innovation et aux avancées technologiques (Kollmuss et Bowell, 2007). La prévention de la déforestation contribue en outre à la conservation de la biodiversité et à la préservation de l'écosystème. La protection de zones de forêts anciennes devrait également être considérée comme un crédit de carbone.

Pour terminer, les voyageurs peuvent prendre les choses en main, au lieu de compter sur les entreprises qui œuvrent dans le secteur des crédits de carbone. Par exemple, quand ceux qui prévoient faire un voyage sont en mesure d'estimer à l'aide d'une calculatrice Internet la quantité d'émissions qu'ils devront compenser. Pour combler cette différence ils doivent alors, avant et après le voyage, soit s'abstenir de conduire leur voiture, soit utiliser des produits ménagers efficaces sur le plan énergétique (Loftus, 2007). Les individus et les entreprises ont déjà la possibilité d'agir pour réduire l'empreinte carbonique associée au voyage. 
Julianna Priskin est professeure associée au Département d'études urbaines et touristiques de I'Université du Québec à Montréal et chercheure à la Chaire de tourisme Transat de la même université.

\section{Renae Nicole Stenhouse est consultante en écologie.}

\section{Note}

1 Dans le présent article, les termes carbone et dioxyde de carbone sont équivalents. Les projets de compensation du carbone visent à compenser les autres gaz à effet de serre. Selon le Protocole de Kyoto, six gaz sont responsables du réchauffement climatique: le dioxyde de carbone, le méthane, l'oxyde nitreux, les hydrofluorocarbones, les perfluorocarbones et l'hexafluorure de soufre (UNFCC, 2007).

\section{Bibliographie}

Anonyme (2007a), «A Special Report on Business and Climate Change », The Economist, vol. 383, n 8531, p. 3-30.

Anonyme (2007b), "Carbon Offsets: Ripping Off Would-be Greens? », The Economist, vol. 382, no 8520.

Australian Greenhouse Office (2006), Investing in Trees as Greenhouse Sinks. An Overview for Industry. A Publication of the Department of the Environment and Heritage, Commonwealth Government of Australia.

Bäckstrand, K., et E. Lövbrand (2006), "Planting Trees to Mitigate Climate Change: Contested Discourses of Ecological Modernization, Green Governmentality and Civic Environmentalism ", Global Environmental Politics, vol. 6, n 1, p. 50-75.

Baral, A., et G.S. Guha (2004), «Trees for Carbon Sequestration or Fossil Fuel Substitution: The Issue of Cost vs. Carbon Benefit ", Biomass and Bioenergy, $\mathrm{n}^{\circ} 27$, p. 41-55.

Barker, R. (2006) «A Special Report on Carbon-Related Issues », New Internationalist, juillet, $\mathrm{n}^{\circ} 391$.

Benitez, P.C., I. McCallum, M. Obersteiner, et Y. Yamagata (2007), « Global Potential for Carbon Sequestration: Geographical Distribution, Country Risk and Policy Implications ", Ecological Economics, vol. 60, n³, p. 572-583.

Brown, K., et E. Corbera (2003), « Exploring Equity and Sustainable Development in the New Carbon Economy ", Climate Policy, vol. 3, n 1, p. 41-56.

Burns, P., et L. Bibbings (2007), Mitigation: Potential for Emission Reduction through Behavioral Change. Policy Dialogue on Tourism Transport and Climate Change: Stakeholders Meet Researchers, Paris, United Nations Educational and Cultural Organization, E-CLAT Technical Seminar, 15 mars.

Gold Standard Foundation, [www.goldstandard.org], (consulté le 11 juin 2007).

Gössling, S., J. Broderick, P. Upham, J. Ceron, G. Dubois, P. Peeters, et W. Strasdas (2007), «Voluntary Carbon Offsetting Schemes for Aviation: Efficiency, Credibility and Sustainable Tourism ", Journal of Sustainable Tourism, vol. 15, n 3, p. 223-248.

Heughebaert, A. (2006), Étude comparative des programmes de compensation volontaire des émissions de $\mathrm{CO}_{2}$ par les passagers d'avions, Bruxelles, Institut de gestion de l'environnement et de l'aménagement du territoire, Université Libre de Bruxelles.

Intergovernmental Panel on Climate Change / IPCC (1996), Technologies, Policies and Mechanisms for Mitigating Climate Change, Geneva, Technical Report 1 of the Second Working Group. Intergovernmental Panel on Climate Change.

Intergovernmental Panel on Climate Change / IPCC (2007), Climate Change 2007: The Physical Science Basis. Summary for Policymakers, Geneva,
Contribution of Working Group I to the Fourth Assessment Report of the Intergovernmental Panel on Climate Change.

Jindal, R. (2006), Carbon Sequestration Projects in Africa: Potential Benefits and Challenges to Scaling Up, Earth Trends, World Resources Institute.

Kelly, J., et P.W. Williams, (2007), «Modelling Tourism Destination Energy Consumption and Greenhouse Gas Emissions: Whistler, British Columbia, Canada ", Journal of Sustainable Tourism, vol. 15, n 1, p. 67-90.

Kirby, K.R., et C. Potvin, (2007), "Variation in Carbon Storage Among Tree Species: Implications for the Management of a Small-scale Carbon Sink Project », Forest Ecology and Management, vol. 246, vol. 2-3, p. 208-221.

Kirschbaum, M.U.F. (2000), «What Contribution Can Tree Plantations Make Towards Meeting Australia's Commitments Under the Kyoto Protocol? ", Environmental Science and Policy, n 3, p. 83-90.

Kollmuss, A., et B. Bowell (2007), Voluntary Offsets for Air-Travel Carbon Emissions. Evaluations and Recommendations of Voluntary Offset Companies, Tufts Climate Initiative, Medford, Massachusetts, Tufts University.

Loftus, M. (2007), "Smart Traveler, It's Not So Simple, Being Green", National Geographic Traveler, vol. 24, n 4, p. 18-25.

Maréchal, K., et W. Hecq (2006), «Temporary Credits: A Solution to Potential Non-permanence of Carbon Sequestration in Forests? ", Ecological Economics, n०58, p. 699-716.

Pearce, F. (2002), «Tree Farms Won't Halt Climate Change », New Scientist, [www.newscientist.com/news/news.jsp?id=ns99992958], (consulté le 28 octobre 2003).

Priskin, J. (2007a), «Le transport aérien. Le voyage à bilan carbone neutre est-il réellement écologique? ", Téoros, vol. 26, n 4, p. 63-66.

Priskin, J. (2007b), « Understanding the Dynamics of Carbon Credit Purchasing from Offsets when Traveling Carbon Neutral ", Tourism Intelligence Network, 22 août.

Richards, K., et K. Anderson (2001), « The Leaky Sink: Persistent Obstacles, to a Forest Carbon Sequestration Program Based on Individual Projects ", Climate Policy, n 1, p. 41-54.

Smith, J., et S.J. Scherr (2003), « Capturing the Value of Forest Carbon for Local Livelihoods ", World Development, vol. 31, n 12, p. 2143-2160.

Sustainable Travel International (2007), Sustainable Travel Report Archives, [www.sustainabletravelinternational.org], (consulté le 3 août 2007).

United Nations Environment Programme / UNEP (2006), Can Carbon Dioxide Storage Help Cut Greenhouse Emissions?, Geneva, Division for Environmental Conventions.

United Nations Framework Convention on Climate Change / UNFCC, [www.unfccc.int], (consulté le 3 août 2007).

United Nations World Tourism Organization / UNWTO (2007), Why Climate Change Matters for Tourism Destinations and Operations?, [http:// www.unwto.org/sustainable/climate.htm], (consulté le 3 août 2007).

Van Kooten, G.C., A.J. Eagle, J. Manley, et T. Smolak (2004), "How Costly Are Carbon Offsets? A Meta-analysis of Carbon Forest Sinks ", Environmental Science and Policy, nº 7, p. 239-251.

Watson, R.T. (2001), The Carbon Cycle: Policy Nexus, Bonn, Presentation by the Chair of the Intergovernmental Panel on Climate Change at the Conference of the Parties to the UNFCCC, 17 juillet.

World Business Council for Sustainable Development/ WBCSD (2007), Completion of the Voluntary Carbon Standard Framework. World Business Council for Sustainable Development News, 31 juillet, [www.wbcsd.org], (consulté le 3 août 2007).

World Resource Institute (2007), [http://www.wri.org/climate/sequestra tion.cfm], (consulté le 3 août 2007). 